\title{
Linking intra-day variations in residential electricity demand loads to consumers' activities: what's missing?
}

\author{
José Luis Ramírez-Mendiola ${ }^{\mathrm{a}, *}$, Philipp Grünewald ${ }^{\mathrm{a}}$, Nick Eyre $^{\mathrm{a}}$ \\ ${ }^{a}$ Environmental Change Institute, University of Oxford,UK
}

\begin{abstract}
An increasing interest in the representation of the intra-day variability observed in domestic electricity consumption patterns has driven the development of various modelling frameworks that incorporate consumer behavioural patterns as a key element for the simulation of electricity consumption. Some of the existing models produce reasonable representations of the broader characteristics of user activity patterns, typically rendered as dwelling occupancy patterns. However, when these activity patterns are used to produce estimates of the electricity demand loads associated with such activities, little attention is paid to the links between activities and the actual use of the electric equipment responsible for the production of the actual demand loads. Instead, the simulation of demand loads from activity patterns is ruled by simplifying assumptions that mask the reality behind those links. This paper therefore seeks to unpack the relationship between activity and electricity demand profiles by focussing on the underlying activity patterns in more detail, and how these relate to the usage patterns of the associated appliances. These relationships are studied based on currently available datasets. The analysis of the activities associated with the use of more than one appliance revealed the differences between the likelihood of each appliance being activated throughout the day relative to user engagement in the activity the appliance is associated with. In practice, what this shows is how each appliance's share of the demand load associated with the activity varies throughout the day. As a result, the power consumption associated with a particular activity is subject to the same kind of variability; activity-related demand. The results of this analysis can be used in conjunction with current or new modelling approaches with a view to linking the user activity patterns with the simulation more of realistic electricity demand loads.
\end{abstract}

(C) 2017 The Authors. This manuscript version is made available under the CC-BY-NC-ND 4.0 licence http://creativecommons.org/licenses/by-nc-nd/4.0/

Keywords: Domestic electricity use, Electricity demand loads, Household activity profiles, Appliance usage

${ }^{*}$ Corresponding author:

Email address: joseluis.ramirezmendiola@ouce.ox.ac.uk,joseluis.ramirezm@hotmail.com (José Luis Ramírez-Mendiola ) 


\section{Introduction}

Daily demand load profiles are becoming increasingly relevant in the planning and design of energy systems. System flexibility is more costly to deliver in low-carbon systems and more detailed information about the intra-day variations in energy demand could therefore afford more cost-effective infrastructure developments.

In countries like the UK, the transition towards a low-carbon economy may lead to the creation of new major demand loads, such as those derived from the electrification of transport and heating [5, 7]. After years of declining electricity demand, which eased capacity constraints, these new loads could pose serious new challenges to capacity provision.

A better understanding of the relationship between user behaviour and electricity demand loads would provide us with an opportunity for improving energy systems' performance. With a more detailed knowledge of the underlying processes that give rise to the observed electricity demand profiles we might be able develop adequate demand-side management (DSM) strategies, which seek to mitigate the need for costly storage or other surplus capacity by shifting the timing and reducing the magnitude of demand [11].

DSM is seen by many as a key component of a smart grid. Robust models, capable of producing accurate representations of energy demand loads and their flexibility in relation to the societal functions they fulfil, are crucial to assess the potential of DSM strategies [24, 31].

The branch of energy demand modelling based on the simulation of user activity is an area of energy research that has recently attracted considerable attention. Several models of residential demand based on occupancy data have been developed over the last decade $[2,13,16,18,20,30,31]$.

The earliest examples have taken a deterministic approach when it comes to linking the occupancy
${ }_{41}$ profiles to the generation of simulated demand loads

42 [31]. The simplicity of this approach yields satisfac-

43 tory results at the aggregate level, but fails to capture the diversity of demand at household level [19]. Probabilistic methods are capable of producing more realistic representations of the variability of metered consumption patterns as demonstrated by [28] and [17].

As Fig. 8(B) illustrates, daily average occupancy would make for a good first order predictor of demand. However, this figure also shows that at some of the most critical times the two measures diverge. Simulations overestimate the morning ramp up and also the duration of the evening peak. Conversely, baseload demand is significantly underestimated.

As has been pointed out elsewhere, any model seeking to represent electricity demand needs to take a detailed account of the timing of energy-related activities [10, 24]. In addition, accurate representations of the timing of the associated demand loads, which result from the use of the associated appliances, plays a vital role in the analysis of scenarios such as the implementation of DSM. The potential future changes in demand make much more relevant to understand the specific appliance use in more detail, as strategies such as load shifting are highly appliance specific [10].

This paper thus aims to help further our understanding of the activity-to-demand dynamics by delving into the links between people's engagement in activities and the (potential) appliance usage that results from it. Section 3 discusses the key elements of the datasets used for the analysis and the data processing methods. A methodology for comparing activity profiles with electrical profiles was developed and is discussed in Section 3.3. We then present activity-specific results and associated appliance use in Section 4.1. The emerging discrepancies and their implications for demand models are discussed in our conclusions section. 
In the residential load modelling literature there is an emerging effort to link electricity demand with the timing of user activities. This approach focuses on simulating household activity patterns, usually through probabilistic methods. These models tend to be based on data from national time-use surveys. A review of models developed based on this approach can be found elsewhere [23].

The release of the data from the UK time-use survey of 2001 [12] motivated the development of one of the most widely implemented models for residential electricity demand based on activity data [19]. This model uses time-use data to simulate daily occupancy profiles which are then used to determine whether certain simulated appliances are activated throughout the day. During states of active occupancy appliance activation is determined based on the probability that members of the household are engaged in the activity the appliance is associated with. The model has been used elsewhere $([17])$ to create a simulated counterpart of an empirical dataset [6]. As the analysis presented in [17] points out, the simulated households were configured such that each one of them had a counterpart in the empirical dataset. That is, parameters such as household size and average household annual consumption were assigned on a one-to-one basis and the appliance contents of the simulated households were matched as closely as possible with those of the corresponding empirical counterpart. As Fig. 1 shows, the model provides a reasonable estimate of the overall average daily load profile. However, as the analysis presented in [17] reveals, the assumptions about appliance usage in relation to engagement in the activities the appliances are associated with lead to a misrepresentation of the associated demand for electricity.

Models based on the same approach, that is, the conversion of active occupancy into demand for electricity, have been developed for other countries,

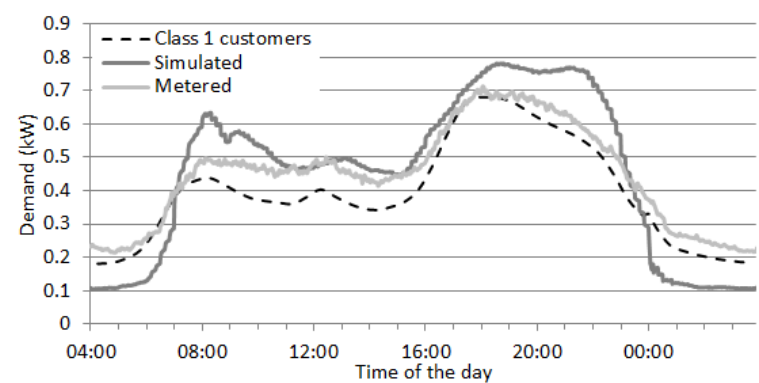

Figure 1: Average daily load profiles. Adapted from [17]. Based on data from $[26,32]$. Class 1 customers refers to ELEXON's customer classification (see [26]). ${ }^{1}$

namely Belgium [1], Spain [14] and Sweden [29]. While there are some differences in terms of the way activity patterns are generated, the assumptions about appliance usage are essentially the same; activity engagement is the only proxy for appliance usage. A more detailed classification of models based on this occupancy-to-demand approach can be found elsewhere [10].

People's engagement in activities is not in itself the cause of the demand loads associated with those activities; rather it is the use of the electrical appliances associated with or needed in order to perform such activities (see Fig. 2). Since the model developed by Richardson et al. [19] was published, detailed datasets on appliance electricity consumption have become available [6]. These new datasets can now be used in conjunction with time-use data to provide new insights into the links between activity patterns and the associated appliance usage.

As has been pointed out in the load modelling literature, small-scale demand modelling needs to account for the influence of household characteristics and the stochasticity of individual behaviours, but it also requires that an accurate representation of the

\footnotetext{
${ }^{1}$ The load profile corresponding to Class 1 customers is depicted to serve as a point of reference to show not only how the simulated and metered profiles compare to one another, but also how they compare to the reference profile. Class 1 comprises residential customers supplied on unrestricted tariffs, with a maximum demand below $100 \mathrm{~kW}$ as measured by metering systems containing only one meter register. There are eight profile classes, and for each profile class a sample group of electricity supply customers is randomly drawn from the population of electricity supply market customers. These samples are designed to provide an accurate estimate of the load pattern for each class of customers for use in electricity settlement processes.
} 
linkage between activity and activity-initiated energy use is achieved [10, 24]. To that end, this paper seeks to help provide a better picture of the activity engagement-appliance usage links so that better representations of them can be realised.

\section{Data and methods}

Brief introductions to data on activities and appliance usage are given in Sections 3.1 and 3.2, respectively. This is followed by a description of the methodology implemented for the extraction of the relevant patterns from the source datasets (Sec. 3.3).

\subsection{Activity data}

Time-use surveys are studies concerned with how people spend their time. Their primary aim is to provide a direct measure of the amount of time spent by the population on various activities; these statistics are collected at a national level. Most EU member states have undertaken this kind of studies since the late 1990s following the guidelines of the Harmonised European Time-Use Study. In 2001, the UK TimeUse Survey (TUS) [15] which has been the base of the most recent time-use studies and activity-based models for the UK was conducted on behalf of several government departments. The surveyed sample comprises 6,414 households from across the UK. All survey respondents were asked to record their daily activities in detailed 24-hour diaries, producing over 18,000 (adult) diary entries. Each respondent was asked to complete diaries for two days: one weekday and one weekend day. Respondents' activities were logged every 10 minutes starting at 4:00 AM on the day in question. The analysis presented in this paper will focus on three key elements of this dataset: main and secondary activity, and location diaries.

The activity information reported in both main and secondary activity diaries is recorded in the form of a pre-defined set of codes which cover a wide range of everyday activities [15]. The two activity diaries combined allow us to get a much better picture of how people spend their time at home; in particular, when they engage in activities that may result in demand for electricity.

Among the surveyed activities, a distinction can be made depending on whether they can be linked to electricity consumption through the use of domestic appliances. For some of those activities it is possible to make a clear distinction. For some others, however, the distinction is somewhat ambiguous. People engaging in these kinds of activities may not necessarily decide/need to make use of appliances. The diagram in Fig. 2 illustrates this point, taking some of the activities surveyed by TUS as examples.

Activity space:

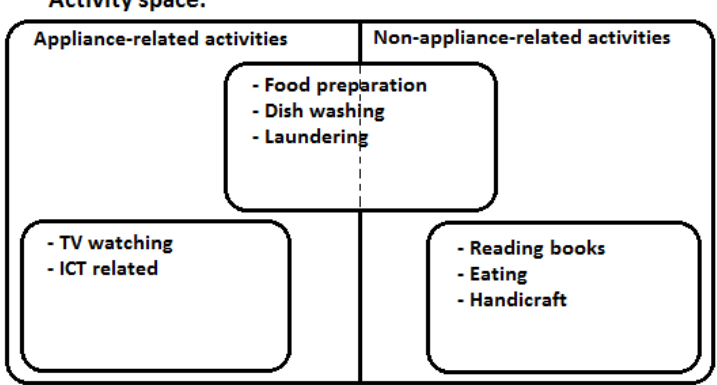

Figure 2: Characterisation of activities according to their relationship with energy use (example).

The activities on which the present analysis focuses are listed in Table 1, along with the monitored appliances that can be (unambiguously) associated with them. A similar mapping between activities and appliances was used by Stankovic et al. [22]. In [22] the authors make use of these associations to draw inferences about the household activity profiles based on metered electricity use. They define an 'activities ontology' that maps a set of 16 activities to the different energy-using devices that can be associated with the corresponding activities. The set of activities is grouped into four broad categories, which include activities corresponding to 'daily routines' and 'computing and leisure'. Within the 'daily routines' categories activities such as cooking, laundering, eating, washing, and sleeping are found. Within the 'computing and leisure' category activities such as watching TV, gaming or using the computer are included. 


\subsection{Appliance usage data}

Over the period May 2010 to July 2011, daily electricity consumption levels of 250 UK households were monitored and recorded as part of a national study [9]. The Household Electricity Survey (HES) was jointly commissioned by Defra, DECC and the Energy Saving Trust and its main aim was to monitor total electricity consumption of the surveyed homes as well as that of individual major appliances [32]. The final report on this study observed that the monitored households were chosen such that the whole sample matched the typical socio-economic mix [32]. Only owner-occupiers were recruited for the study. The households, however, were still fairly typical in terms of socio-demographic factors [32].

The data from this study [6, dataset] allow us to identify overall daily appliance usage patterns. The dataset is a collection of daily load profiles corresponding to the appliances monitored within the surveyed households. The overall length of the monitoring period of each appliance varies across households; some households were monitored for a full year, whereas others were monitored for "one-monthlong" periods with effective lengths varying between 20 and 30 days.

The monitored appliances can be grouped into categories corresponding to the groups of appliances typically used when performing certain activities (Table 1). There are also some appliances which could be broadly categorised as non-activity-related energy uses (e.g. cold appliances). Despite the fact that these appliances remain important in terms of their energy use, they are not significantly affected by the activities of the members of the household. As this study focuses on exploring the linkages between user activities and appliance usage, this kind of appliances was excluded from the analysis.

Table 1 summarises the areas in which there are reasonable expectations that a mapping between user activities and monitored energy use exists. It lists the activities that are studied in this paper, as
Table 1: Activities and list of monitored appliances associated with them.

Activities Associated appliances

(from TUS diaries) (monitored by HES)

\section{Food preparation} cooker, oven, microwave, kettle

\begin{tabular}{cl}
\hline \multirow{2}{*}{ Laundering } & $\begin{array}{l}\text { Washing machine, } \\
\text { washer dryer, Tumble } \\
\text { dryer }\end{array}$ \\
\hline Dish washing & Dish washer \\
\hline \multirow{3}{*}{ TV watching } & $\begin{array}{l}\text { TV1, TV2, TV3, CRT } \\
\text { TV, LCD TV, plasma } \\
\\
\text { TV, Audio-visual site }\end{array}$ \\
\hline \multirow{2}{*}{ ICT related } & Desktop PC, \\
& Computer site, Laptop \\
\hline
\end{tabular}

As we observed in the previous section, the mapping of appliances onto activities defined in this paper is consistent with the one used in [22]. However, we focus on those activities that are meaningful in terms of households' daily routines and likely to have a significant share of total electricity consumption. The appliances associated with the activities analysed in this paper account for over $40 \%$ of the total household annual consumption [4]. Therefore, there is great potential for the use of these appliances to reduce both daily and annual household electricity consumption. However, in order to provide a good estimate of such potential, and more importantly to be able to exploit it, it is necessary to have a better understanding of the way these appliances are used and how their use relates to the reported activity patterns.

There are many other activities that may result in demand for electricity. However, these activities may not necessarily be a determinant in the everyday lives of households. Therefore, identifying their corresponding electricity demand loads based on currently available data would prove considerably more complicated. In this context, we refer to an activity 
as a 'determinant' in the daily routines when there is a reasonable expectation that this kind of activity is likely to be of high priority. That is, some 'less important' activities are likely to be planned around those high-priority activities, and this in turn would determine the overall daily routine/activity pattern. There is always a possibility that this kind of activities may in fact be determinant of the overall daily activity patterns of certain individuals or groups of people. However, based on what is observed in the empirical data, this is rarely the case and therefore neglected.

\subsection{Extraction of daily activity and appliance usage patterns}

Activity and appliance usage data stem from different studies undertaken at different times. However, meaningful comparisons can be made between them focusing on the generic links. In order to do this, two assumptions are made. First, it is assumed that the patterns observed in these two samples are indeed representative of the overall patterns across the broader population; the second assumption is that the potential changes in the timing of activities and appliance usage during the time gap that separates both studies were small enough so as to have the overall patterns preserved.

Daily activity profiles were extracted from the time-use data for each of the activities listed in Table 1. At any given $10 \mathrm{~min}$ interval of the day, the rate of engagement in a particular activity is given by the number of times TUS respondents reported engaging in that activity at that time over the total number of diary slots in TUS records for the corresponding interval. In other words, it is the probability that a random person would engage in this activity at this time of the day. To produce the activity profiles, a combination of main and secondary activity diaries was used. The need for the use of the two diaries is due to the fact that some activities with a very direct link to electricity consumption, such as TV watching, are often deemed to be secondary activities. In order to account for this possibility, both diaries are analysed simultaneously. In this way, it is ensured that the most accurate representation of people's engagement in certain activities throughout the day is achieved. The activities recorded in the activity diaries are by no means restricted to a particular location. Therefore, we made use of the location diaries to filter for "in home" activities.

In addition, based on the activities and locations reported in these diaries, overall occupancy profiles were also extracted. These occupancy profiles essentially show the probability of finding a random person engaging in any of three occupancy states considered: active occupancy, passive occupancy (sleeping) and absence. Based on each individual adult diary, an occupancy profile featuring the three occupancy states previously mentioned was created. Then, in a similar manner to the calculation of the activity profiles, the overall occupancy profile was calculated by aggregating all the individual profiles.

Daily appliance usage profiles were extracted from the HES data for each of the appliance groups listed in Table 1. The HES dataset is basically a collection of daily appliance-level electricity consumption profiles. Therefore, a direct comparison against the TUS data is not possible. The methodology for the extraction of the appliance usage profiles is different to the one used for TUS data processing, as this involved a more complex treatment of the relevant data. The main goal was to determine when an appliance was being actively used; hence, any baseload-like contributions to the load profile had to be discarded.

For the treatment of HES data two things had to be taken into consideration. Firstly, the length of the monitoring period of each appliance varies, due to the issues discussed in Sec. 3.2. What this means in terms of the analysis is that the number of days each kind of appliance (e.g. "kettle") was monitored across households is different. For this reason, the monitoring period of each individual appliance had 
to be treated separately.

Secondly, appliances found in different households have different consumption characteristics, in spite of sharing a common label in HES records. That is, appliances with the same label may have different power requirements, different duty cycles, standby consumption and so on. Based on all the available data on each of the monitored appliances, the baseload consumption of each appliance (i.e. standby or losses) was estimated. This was then used as a threshold value for determining the moments of the day in which the appliance was actually in use. An appliance is considered to be "on" when its stand-by consumption threshold is exceeded by $50 \%$. This is an extra precaution to account for the fluctuations observed in the power drawn during the stand-by phases of the different appliances. It is not possible to determine whether these fluctuations are metering errors or actual variations in the power drawn by the appliance. However, it is still necessary to account for them in order to avoid false positives.

A daily usage profile was thus generated based on this information for each day of the monitoring period of that particular appliance. These usage profiles are binary sequences, where 1 indicates an active state and 0 a state of inactivity. All the daily usage profiles corresponding to appliances sharing the same label are then aggregated. At any given 10 min interval, the rate of use of a particular appliance is given by the number of times the appliance was being actively used at that particular time over the total length of the monitoring period of that particular appliance in HES records. In other words, it is the probability of finding that type of appliance in use at that time of the day.

The purpose of these profiles is to provide a graphic summary of the likelihood of finding a given appliance in use at a given time of day. Although based on metered appliance electricity consumption data, the profiles should not be taken as probabilistic models for appliance usage as such. The data used for the analysis could also be used to create a simple probabilistic model of appliance usage by assuming, for instance, a first order Markov process. To this end, transition probability matrices would need to be extracted for each transition between the time intervals into which the day is divided. For the purpose of the analysis presented in this paper, however, the likelihood profiles presented are sufficient.

For the activities associated with more than one type of appliance, in addition to individual appliance usage profiles, general usage profiles based on the use of all the appliances associated with the activity were extracted. These profiles are meant to provide an estimate of users' engagement in these activities based on the use of the associated appliances. The data processing for this part of the analysis was carried out in a similar manner to that of the individual appliance usage profiles; this time, however, taking all the relevant appliances into account. For example, to extract the general cooking appliances' usage profile, daily usage profiles were obtained for every day in the monitoring periods of every cooking appliance. Then, the general appliance usage profile was derived from the overall aggregate of daily usage profiles and the total number of days in the combined monitoring periods.

By using these profiles we are able to compare the two datasets in terms of activity and appliance use probabilities. In the following section we present the results of such comparisons.

\section{Results and Discussion}

Activity and appliance usage profiles were extracted from the TUS and HES data respectively in order to explore the links between activity and activity-related energy use. First, we present the results of the analysis of the five activities of interest and their corresponding appliances' usage profiles, and discuss the observed relationships. Next, we use the average occupancy profile extracted from 
the time-use data to show how the oversimplification of these linkages and the influence that active occupancy has over electricity demand loads may lead to severe misrepresentations when simulating electricity consumption.

\subsection{Activity profiles and appliance usage}

As we discussed in Section 3, the activities analysed as part of this study were chosen based on: (1) their general importance in terms of the representation of residential users' behavioural patterns, and (2) the reasonable expectation that such activities could results in electricity use. Table 1 summarises the activities relevant to this study, as well as the monitored appliances which were associated with them for the purpose of the analysis.

In the sections below activity-specific results are presented and discussed.

\subsubsection{Food preparation/Cooking}

A common general pattern is observed in the food preparation activity profile and the general cooking appliances' usage profile derived from all the relevant appliances in HES data; as Fig. 3(A) shows, three peaks approximately centred around mealtimes can be identified. Two main differences between these two profiles can be pointed out. In the general appliances' usage profile the evening peak occurs about 40 minutes later than in the activity profile. Moreover, this general appliances' usage profile would appear to indicate that engagement in cooking activities throughout the day is more likely than the activity profile would suggest.

The differences in the likelihood levels are particularly noticeable in the period between 7:00 and 15:00 hr. The higher levels, however, are not necessarily an indication that the amount of people engaging in cooking activities during this time is larger. These discrepancies are rather due to the fact that simultaneous use of cooking appliances appears to be more common during daytime. This could potentially be decline

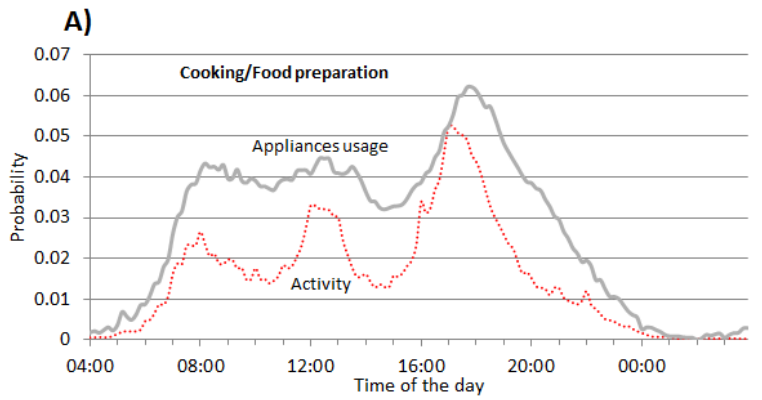

\section{B)}

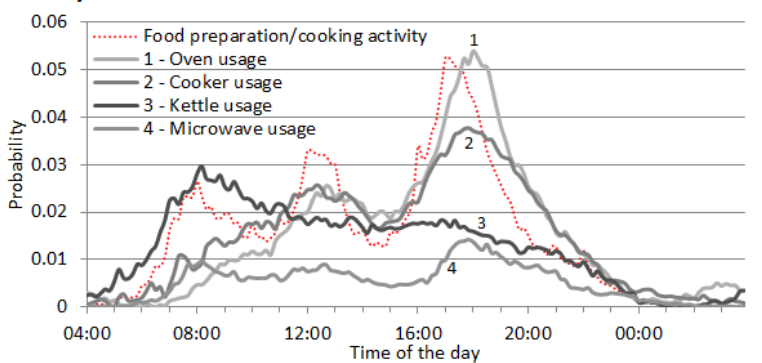

Figure 3: Daily food preparation and cooking activity profiles. A) General cooking appliances' usage profile. B) Individual cooking appliances' usage profiles.

attributed to people making preparations for meals in advance. Fig. 3(B) shows that as dinner time approaches and the final stages of the day's cooking or food preparation activities get under way the use of ovens and cookers ramps up, whereas the use of the other smaller appliances (e.g. kettles) continues to

The usage profiles corresponding to the four main cooking appliances in HES records are plotted alongside in Fig. 3(B). These are also the appliances primarily associated with cooking activities in a more general context. Their corresponding usage profiles clearly show that the likelihood of each one of these appliances being used throughout the day varies at different rates. The comparison of individual usage profiles makes evident that the contribution to the overall demand load of each one of the cooking appliances varies independently throughout the day. What this could mean in practice is that the 'relative usefulness' of each appliance changes depending on the time of the day. For instance, it is observed that food preparation activities in the morning appear to be strongly correlated with the use of kettles; the use of the other appliances appears to be much less relevant in comparison. In contrast, in the evening, the 
correlation between food preparation activities and the use of ovens appears to be the strongest.

\subsubsection{Laundering}

Laundering activities appear to be more prominent in the morning hours. Fig. 4(A) shows that there is a major peak in both laundering activity and appliance usage centred around 10:30 hr, followed by a steady decline towards the end of the day. As in the case of food preparation activities, a general common pattern is observed in both activity and appliance usage profiles. Two main differences between activity and appliance usage profiles stand out. Both profiles follow each other very closely up until the peak occurs. However, after the peak the general appliances' usage profile would appear to indicate that engagement in laundering activities is more likely than the activity profile would suggest. In addition, the general appliances' usage profile would appear to indicate that there is a second peak, approximately centred around 19:00 hr. The higher likelihood levels observed in the general appliances' usage profile could potentially be attributed to the differences between the perceived duration of laundering activities from a user perspective and the actual duration of an appliance's cycle of operation. Users would only report being engaged in laundering activities when they are actively taking part in the process; loading and activating a washing machine, for instance. From the appliance's perspective, however, laundering activity would be registered from the moment the appliance is activated and until its duty cycle is completed.

As Fig. 4(B) shows, the individual usage profiles corresponding to washing machines and tumble dryers are essentially different. The peak in washing machines' usage is consistent with the peak observed in the laundering activity profile; both peaks occur roughly at the same time of day. Washing machine usage follows the same general pattern observed in the activity profile. However, the appliance usage profile shows consistently higher levels of likeli-
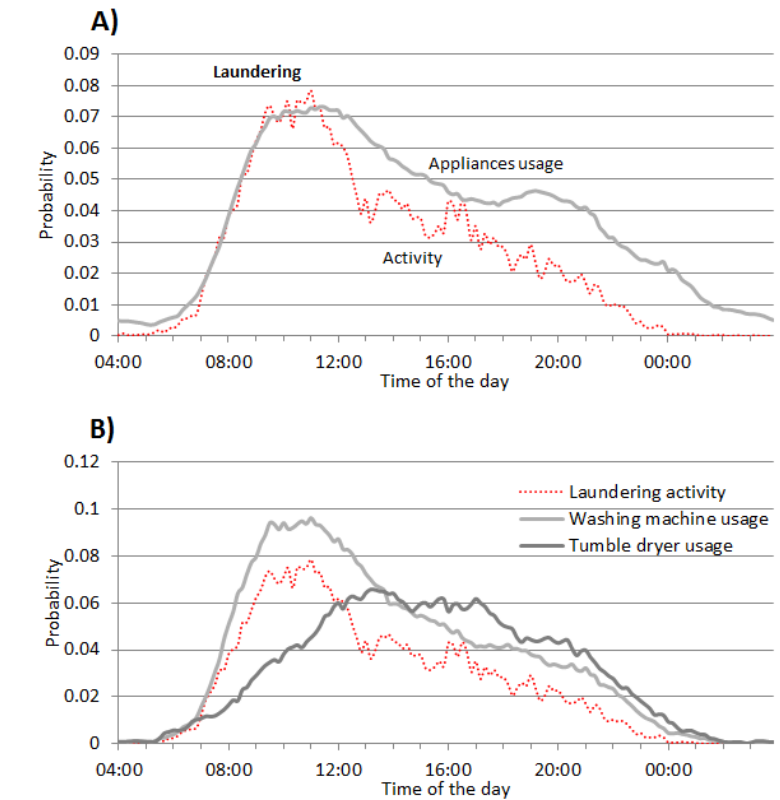

Figure 4: Daily laundering activity profile. A) General laundering appliances' usage profile. B) Individual laundering ap-

pliances' usage profiles.

hood. The observed differences can be attributed to the same phenomenon observed in Fig. 4(A). Tumble dryers' usage profile shows a different trend to the one observed in the laundering activity profile. Rather than presenting a well-defined peak in the morning, this profile looks more like a plateau with a maximum likelihood period extending approximately from 13:00 to 17:00 hr. This period is followed by a gradual decline towards the end of the day, consistent with what is observed in both the washing machine usage and activity profiles.

The differences between the washing machine and tumble dryer usage profiles appear to be consistent with what is observed in practice. Firstly, they reflect the fact that when tumble dryers are used they are almost invariably run after washing machines. Secondly, the generally lower rates of use reflected by the tumble dryer usage profile can also be attributed to the fact that tumble dryers are less ubiquitous than washing machines. In addition, tumble dryers are less commonly used due to the use of other drying methods (e.g. line drying).

\subsubsection{Dish washing}

The dish washing activity profile shows three well defined peaks, clearly centred around typical post- 
prandial times. The dishwasher usage profile, however, as Fig. 5 shows, appears to be divided into two broader regions: before and after 16:00 hr. The first region exhibits a single peak that is consistent with the morning peak observed in the activity profile. However, the peak in the appliance usage profile occurs about 40 minutes later than in the activity profile. During the period when the midday peak occurs in the activity profile, the usage profile exhibits a more levelled region. As for other appliances, this could be attributed to the effect of the duration of the appliance's cycle of operation.

The second region of the dishwasher usage profile exhibits two peaks. The first one is consistent with the evening peak observed in the activity profile. As the morning peak, this peak in appliance usage occurs about $1 \mathrm{hr}$ later than in the activity profile. Figure 5 also suggests that there is a strong tendency among dishwasher owners to run the appliances overnight, as the peak occurring around midnight indicates.

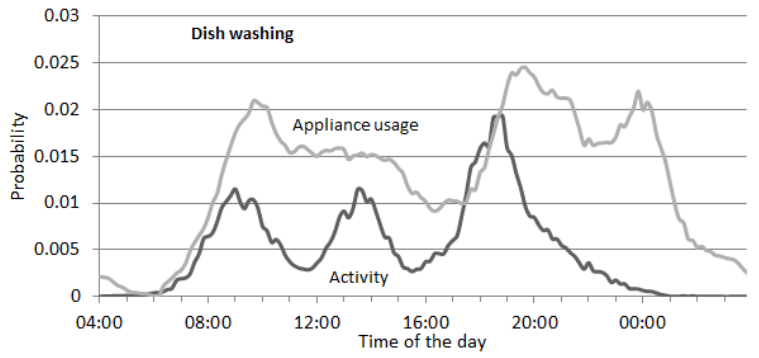

Figure 5: Daily dish washing activity and metered dishwasher usage profiles.

\subsubsection{TV watching \& ICT related}

Perhaps not surprisingly, TV watching is most prominent in the evening hours. However, Figure 6(A) shows that reported TV watching is significantly lower than the measured consumption from TV sets would suggest. In the first instance, this can be attributed to the systematic under-reporting of the time spent watching TV. It may, however, also be related to the definition of the activity itself.

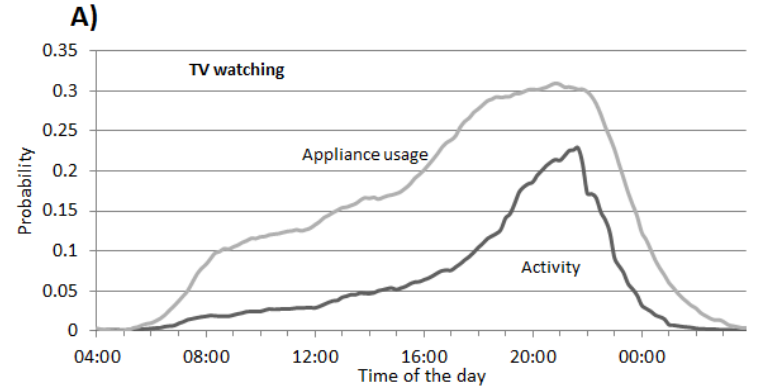

B)

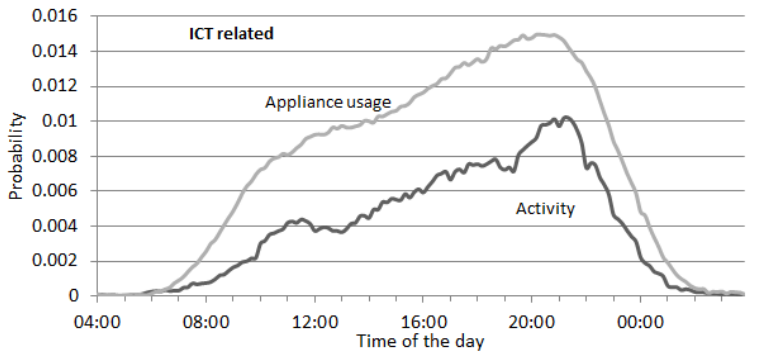

Figure 6: Daily TV watching and ICT related activity profiles. A) TV watching activity and metered overall TV usage profiles. B) ICT related activity and metered overall IT appliances' usage profiles.

Participants in time-use surveys are asked to report on what they do, not which appliances are in use. A TV set, which is permanently running in the background while people go about their lives, will not be reported as an activity. Figure 6(A) suggests

that this under-reporting effect is greatest during the daytime (up to $70 \%$ ), whereas the evening peak at around $10 \mathrm{pm}$ is captured fairly accurately in time and also better captured in magnitude. The lower under-reporting at peak $(25 \%)$ could be a result of more people 'actively' watching TV.

\subsection{Dependence of simulated power consumption on} active occupancy

Previous studies have pointed out that the daily electricity load profile is strongly affected by changes in the occupancy of dwellings throughout the day. A combination of the activity and location diaries from TUS data allows us to determine the proportion of people likely to be engaged in a certain occupancy state at each $10 \mathrm{~min}$ interval of the day. The resultant average daily residential occupancy profile has a distinctive shape that translates into clear indications about people's preferences when it comes to where they spend their time at different times of day. 
These preferences, of course, are subject to some change; the differences in these preferences manifest themselves, for instance, when the average occupancy profiles corresponding to weekdays and weekend days are compared (see Fig. 7). In each of these profiles, three shaded regions can be observed. Each one of those regions corresponds to one of three basic occupancy states: active occupancy, passive occupancy (sleeping) and absence. The vertical axis indicates the probability of finding a random person engaging in any of the three states considered.

The differences in the typical everyday activities for the two types of days are evident from these profiles. As Fig. 7 shows, despite the daily occupancy profiles having roughly the same shape, the probabilities of finding a random individual at home at a given time of the day differ. For instance, on average, it is about $20 \%$ more likely to find a random person at home between 9:00 and 15:00 hr on weekend days than on weekdays. These differences also provide a measure of people's tendency to get up later on weekends and stay in longer. Figure 7(A) shows that on weekdays there is a short, sharp morning peak centred around 8:00 hr. The period leading to the observed peak is consistent with the typical transition period from being asleep to being active, preparing for the start of the day. The peak is followed by a sharp decline, corresponding to the sudden change in occupancy that occurs when people leave home for work, school, or any other activities taking place at a different location. In contrast, Fig. 7(B) shows that on weekend days the transition from being asleep to being active appears to be less sharp, with the peak in active occupancy showing up about 90 minutes later than in weekdays. Moreover, the decline in the likelihood of finding a random person asleep as the morning progresses is more gradual during weekends.

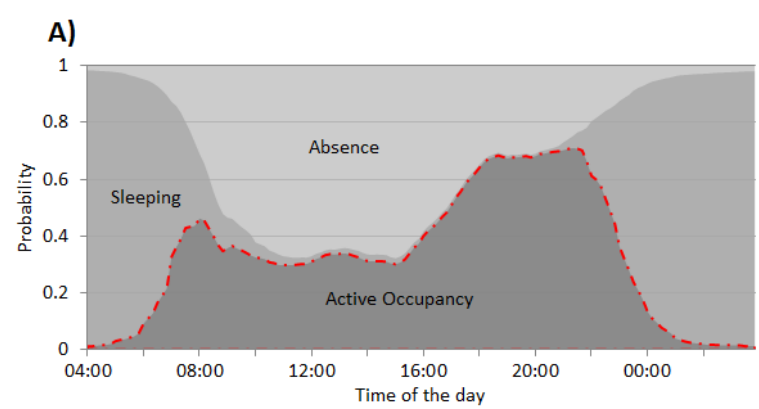

B)

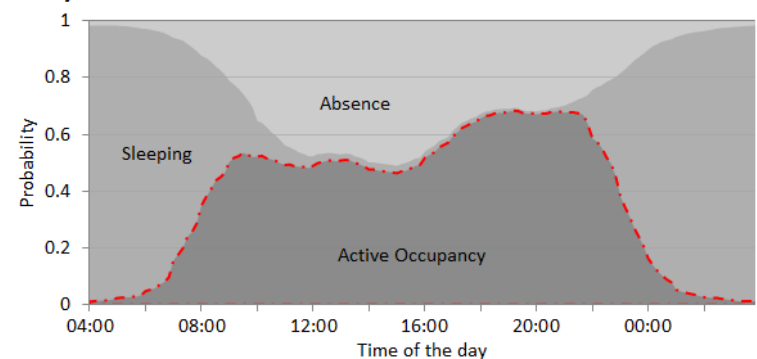

Figure 7: Average occupancy: A) Weekday average occupancy profile. B) Weekend average occupancy profile. The different shaded areas represent, at any given 10 min interval, the overall probability that an individual is in one of three occupancy states: Active at home, sleeping or absent.

The times at which the evening peak is observed are also different. In both types of days, the likelihood of finding a random individual active at home gradually increases as the evening approaches, arriving at a peak of about $70 \%$. On weekdays the peak is observed from 18:00 to 21:30 hr, whereas on weekend days it extends from 19:00 to 22:00 hr. Towards the end of the day, the decline in the likelihood of finding a random individual active is accompanied by a considerable increase in the probability of finding them asleep in both profiles.

The differences observed between the average occupancy profiles corresponding to the two types of days are relevant for a more detailed analysis of the daily occupancy patterns or for the refinement of occupancy models. These differences also have effects in terms of the observed electricity consumption, of course. For the purpose of illustrating and discussing the relationship between occupancy and the daily electricity demand load profile, however, a comparison between the overall average occupancy profile (Fig. 8(A)) and the average electricity load profile (Fig. 8(B)) will suffice. 


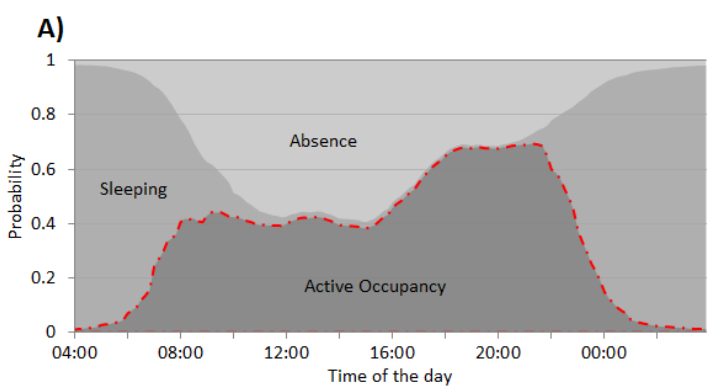

B)

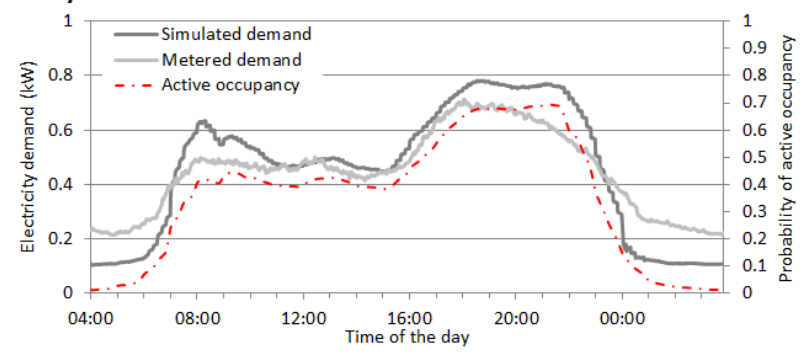

Figure 8: A) Average occupancy profile. B) Comparison between overall active occupancy profile and average daily load profiles.

In Fig. 8(B) metered and simulated load profiles, and average occupancy profile are superposed to allow for an easier contrast. In this figure the strong correlation between active occupancy and demand for electricity can be clearly appreciated. Clearly, daily average occupancy would make for a good first order predictor of demand. That is, a reasonable estimate of the overall daily load profile could be obtained by simply multiplying the average occupancy (a dimensionless quantity) by a linear (first order) power consumption factor. However, it can also be observed that the strength of this correlation varies throughout the day.

To further discuss this, let us divide the load profiles into three regions characterised by the level of demand: lowest, medium, and highest demand. If the duration of these periods is compared pairwise, it is observed that the durations of the first two regions are very similar in both metered and simulated load profiles. Incidentally, this also seems to be in agreement with the duration of the equivalent periods in the active occupancy profile. However, looking in more detail at the region corresponding to the evening peak, discrepancies can be observed in terms of the duration of this period in the different profiles.

As Fig. 8(B) shows, the duration of the peak period of the simulated demand profile is virtually the same as the duration of the active occupancy peak. In contrast, in the metered demand profile, the duration of the period during which the maximum demand is held is roughly half as long as in the simulated profile.

A related issue has to do with the over-estimation of the influence that active occupancy has over demand loads. A potential indicator of this can be identified by noting that the shape of the simulated load profile follows more closely the shape of the average daily occupancy profile than that of the metered profile. Furthermore, demand levels, especially during peak periods, are over-estimated in the simulated load profile. Special attention should be paid to the most energy intensive appliances, such as those typically used for cooking. The over-representation of the use of one or more of these appliances can result in the kind of effects observed in the simulated profile used as example here. For instance, the simulated profile in Fig. 8(B) shows a morning peak considerable higher than the one observed in the metered profile. This would appear to be consistent with an overrepresentation of the appliances that, as we showed in the previous section, are not as heavily used as in other times of the day.

\subsection{Activity and activity-related consumption pat-} terns in the literature

Over the last decades, several studies on time-use data have provided us with some insight into the ways people go about their lives. As discussed in Section 2, this kind of studies has also provided opportunities for the development of models that seek to capture the stochasticity observed in the empirical behavioural data. While the simulation of activity patterns appears to have become the focus of attention for this kind of studies, the link between engagement in activities and actual generation of electric loads, that is, appliance usage, seems to be largely 
neglected.

Few attempts have been made to better understand the activity-related electricity consumption $[3,8,21,25,27]$. However, these studies have focused on the analysis of electricity consumption aggregates over certain periods of time (e.g. day, month, year), rather than on the intra-day variations. This has probably been largely due to the lack of highresolution appliance-level consumption datasets. In addition, these studies have also focused on single activities associated with single appliances.

For instance, the analysis presented by Torriti and Santiago [25] focuses on an activity in particular: TV watching. The authors used a very simplistic model to produce estimates of aggregated electricity demand based on the number of households potentially engaged in TV watching at any given time of day. As in the other modelling examples (Sec. 2), the model used in [25] assumes that activity data on TV watching, or any other single-appliance activity for that matter, is good enough a proxy for the activity-related electricity demand so that it can be used to estimate demand with a simple linear model. As the results of the analysis presented in this paper clearly show (See Section 4.1.4), this is not the case. There are effects that simplistic linear models cannot account for, such as the observed background TV usage which can be inferred from the metered TV usage. As we discussed in section 4.1.4, both the activity engagement and appliance (TV) usage profiles resemble each other in terms of overall shape. However, by comparing the two profiles it becomes evident that the data on TV watching activities is not enough in itself to produce an accurate estimate of the metered TV electricity consumption.

In the study carried out by Anderson [3], the daily laundering activity patterns extracted from time-use data from different years (1985 and 2005) were compared. The aim of this study was to analyse the evolution of the laundering activities throughout the years, hoping that this would reveal ways in which

\footnotetext{
${ }^{2}$ HETUS - Participant countries: Italy, Spain, Estonia, Latvia, Lithuania, Poland, Bulgaria, Slovenia, UK, France, Germany, Belgium, Finland, Norway and Sweden.
} more importantly, ways in which these could be modified to achieve specific outcomes, which is one main aims of the DSM strategies.

The analysis revealed that there have been significant changes in terms of the days of the week during which laundering activities take place. However, as the author himself points out, whilst the changing distribution of laundry across the week offers insights into the evolving nature of laundry overall, it is the nature of its timing within the day which is likely to be of greater concern in the DSM context.

In addition to the relevance of the timing of engagement in laundering activities, the results for the associated appliance usage presented in this paper (See Section 4.1.2) point out the need to account for the fundamentally different usage patterns observed for washing and drying machines in relation to engagement in laundering activities.

\subsection{Limitations and further research}

Due to the nature of the kind of studies from which the data used for the analysis stems, the results of the analysis are by definition country-specific. While there are some clear limitations to the data used for the analysis presented in this paper, the results provide a number of insights into the relationship between engagement in activity and actual appliance usage.

Activity patterns are, in general, different in different countries and therefore, it only makes sense to compare them to consumption patterns from the same country. However, it has been pointed out elsewhere that there is a high level of similarity between the active occupancy profiles of the 15 European countries that have taken part in the Harmonised European Time-Use Survey (HETUS) ${ }^{2}$ [25]. It is therefore natural to expect that there might be some

practices in the residential sector have changed, and 
similarities in the activity patterns, and even perhaps in the associated appliance usage as well.

Given the relevant data, i.e. large enough appliance-level electricity consumption datasets, similar analyses could be carried out for different countries, which would provide opportunities for further discussion of country-specific results in terms of the discrepancies or similarities observed across countries, and what these entail.

What this study tries to point out is the need to refine our assumptions about activity-related appliance usage in the context of electricity demand modelling in the residential sector. As the results of the analysis show, even appliances associated with the same activity may have completely different daily usage profiles, which is something that models developed to date have failed to take into account. Whatever the reasons might be for the overlooking of these dynamic links between engagement in activities and appliance usage, the results of the analysis presented in this paper show that we are now in a position to better represent these links, and therefore, generate better estimates.

\section{Conclusions}

In this paper, the links between residential consumers' behavioural patterns and appliance usage patterns were explored to improve our understanding of the intra-day variations in residential electricity demand loads.

We presented a methodology for the extraction and comparison of probability profiles derived from time-use data and appliance-level electricity consumption.

The analysis of the discrepancies between these two profiles allows us to challenge commonly held assumptions about the relationship between activities and electricity consumption.

Four types of activities with high relevance to energy consumption are presented in detail. These are food related, laundering, dish washing and TV watching.

The energy intensity of food preparation varies throughout the day. Both the number of appliances involved in food preparation activities and the level of simultaneous use change depending on the time of the day. One therefore cannot use the activity 'food preparation' as the only proxy for electricity consumption, irrespective of the time of day. The analysis further shows that the type of appliances predominantly in use varies throughout the day. The use of high power/low energy demanding kettles is predominant in the morning, whereas evenings see a predominant use of ovens, which entails significantly higher energy consumption.

Dish washing is a good example for time shifts between activity and appliance usage. The reported activity follows an expected pattern featuring peaks after morning, midday and evening meals, with evening dish-washing being twice as common as daytime. Dishwasher usage does not follow this pattern. The peaks in appliance usage occur later than those in the reported activity, which is to be expected, since 'starting the dish-washer' will lead to consumption later on. Yet, at midday this sequence is not preserved. Instead the dish-washer cycle appears to be delayed until after the evening meal, and some of this is delayed even further to operate at the end of the day.

Laundering appliances' usage has the same 'delay effect' as dish-washers', but the intra-day activity pattern is a more reliable indicator of the link between the two. The strong sequencing of washing machine use and tumble drying is evident from the data.

TV watching revealed potentially systematic shortcomings in the link between reported activities and appliance usage. We conclude that a combination of under-reporting of TV watching and inactive use of TVs in the background both lead to a profound gap between activity and appliance usage data 


\section{Acknowledgements}

The authors gratefully acknowledge funding from: the Consejo Nacional de Ciencia y Tecnología (CONACyT), and the Engineering and Physical Sciences Research Council (EPSRC) Ref. $\mathrm{EP} / \mathrm{M} 024652 / 1$

\section{References}

[1] Aerts, D., Minnen, J., Glorieux, I., Wouters, I., Descamps, F., may 2014. A method for the identification and modelling of realistic domestic occupancy sequences for building energy demand simulations and peer comparison. Building and Environment 75, 67-78.

URL https://doi.org/10.1016/j.
[2] Andersen, P. D., Iversen, A., Madsen, H., Rode, C., 2014. Dynamic modeling of presence of occupants using inhomogeneous Markov chains. Energy and Buildings 69, 213-223.

URL https://doi.org/10.1016/j.enbuild. 2013.10 .001

[3] Anderson, B., 2016. Laundry, energy and time: Insights from 20 years of time-use diary data in the United Kingdom. Energy Research and Social Science 22, 125-136.

URL http://doi.org/10.1016/j.erss . 2016.09 .004

[4] BEIS, 2016. Chapter 3: Domestic energy consumption. In: Energy Consumption in the UK. Department of Business, Energy and Industrial Strategy, London, United Kingdom, Ch. 3, p. 17 .

URL https://www.gov.uk/government/ statistics/energy-consumption-in-the-uk

[5] CCC, 2008. Building a low-carbon economy - the UK's contribution to tackling climate change. The Stationary Office, London, United Kingdom.

[6] DECC, Intertek Testing and Certification Ltd., 2016. Household Electricity Survey, 2010-2011. [data collection]. UK Data Service. SN: 7874. URL http://doi.org/10.5255/UKDA-SN$7874-1$

[7] Department of Energy and Climate Change, 2011. The Carbon Plan: Delivering our low carbon future. Tech. Rep. December, Department of Energy and Climate Change, London, United Kingdom.

[8] Durand-Daubin, M., nov 2013. Household activities through various lenses: crossing surveys, diaries and electric consumption. In: Behav. Energy Clim. Chang. Conf. 2013. Berkeley, CA, US. 
URL http://escholarship.org/uc/item/ 83z390h2\{\\#\}page-1

[9] Energy Saving Trust, DECC, DEFRA, Owen, P., 2012. Powering the nation: Household electricity-using habits revealed. Tech. rep., Energy Saving Trust, Department of Energy and Climate Change, Department for Environment Food and Rural Affairs, London, United Kingdom.

[10] Flett, G., Kelly, N., 2017. A disaggregated, probabilistic, high resolution method for assessment of domestic occupancy and electrical demand. Energy and Buildings 140, 171-187.

URL http://doi.org/10.1016/j.enbuild. 2017.01 .069

[11] Haney, A. B., Jamasb, T., Platchkov, L. M., Pollit, M. G., 2011. Demand-side management strategies and the residential sector: lessons from the international experience. In: The Future of Electricity Demand: Customers, Citizens and Loads. Cambridge University Press, Ch. 14.

[12] Ipsos-RSL, Office for National Statistics, 2003. United Kingdom Time Use Survey, 2000 [computer file]. 3rd Edition. Colchester, Essex: UK Data Archive [distributor], SN: 4504,.

URL http://doi.org/10.5255/UKDA-SN4504-1

[13] Lee, Y. S., Malkawi, A. M., 2014. Simulating multiple occupant behaviors in buildings: An agent-based modeling approach. Energy and Buildings 69, 407-416.

URL https://doi.org/10.1016/j.enbuild. 2013.11 .020

[14] López-Rodríguez, M., Santiago, I., TrilloMontero, D., Torriti, J., Moreno-Munoz, A., nov 2013. Analysis and modeling of active occupancy of the residential sector in Spain: An indicator of residential electricity consumption. Energy Policy 62, 742-751.

URL http://doi.org/10.1016/j.enpol. 2013.07 .095

[15] ONS, 2003. The United Kingdom 2000 Time Use Survey: Technical Report. Tech. rep., Office for National Statistics, London, United Kingdom.

URL https://discover.ukdataservice.ac. $\mathrm{uk} / \mathrm{series} /$ ?sn=2000054

[16] Page, J., Robinson, D., Morel, N., Scartezzini, J.-L., 2008. A generalised stochastic model for the simulation of occupant presence. Energy and Buildings 40 (2), 83-98.

URL https://doi.org/10.1016/j.enbuild. 2007.01 .018

[17] Ramírez-Mendiola, J. L., Grünewald, P., Eyre, N., 2017. The diversity of residential electricity demand - A comparative analysis of metered and simulated data. Energy and Buildings 151, 121-131.

URL https://doi.org/10.1016/j.enbuild. 2017.06 .006

[18] Richardson, I., Thomson, M., Infield, D., jan 2008. A high-resolution domestic building occupancy model for energy demand simulations. Energy and Buildings 40 (8), 1560-1566.

URL https://doi.org/10.1016/j.enbuild. 2008.02 .006

[19] Richardson, I., Thomson, M., Infield, D., Clifford, C., 2010. Domestic electricity use: A highresolution energy demand model. Energy and Buildings 42 (10), 1878-1887.

URL https://doi.org/10.1016/j.enbuild. 2010.05 .023

[20] Robinson, D., Wilke, U., Haldi, F., 2011. Multi agent simulation of occupants' presence and behaviour. In: Proc. Build. Simul. 2011 12th Conf. 
Int. Build. Perform. Simul. Assoc. pp. 2110 2117 .

[21] Sekar, A., Williams, E., Chen, R., jun 2016. Heterogeneity in time and energy use of watching television. Energy Policy 93, 50-58.

URL http://doi.org/10.1016/j.enpol. 2016.02 .035

[22] Stankovic, L., Stankovic, V., Liao, J., Wilson, C., 2016. Measuring the energy intensity of domestic activities from smart meter data. Applied Energy 183, 1565-1580.

URL https://doi.org/10.1016/j. apenergy.2016.09.087

[23] Torriti, J., 2014. A review of time use models of residential electricity demand. Renewable and Sustainable Energy Reviews 37, 265-272.

URL https://doi.org/10.1016/j.rser. 2014.05 .034

[24] Torriti, J., mar 2017. Understanding the timing of energy demand through time use data: Time of the day dependence of social practices. Energy Research and Social Science 25, 37-47. URL http://doi.org/10.1016/j.erss . 2016.12 .004

[25] Torriti, J., Santiago, I., jul 2016. Simultaneous activities in the household and residential electricity demand in Spain. Time and Society. URL http://doi.org/10.1177/ $0961463 X 16656867$

[26] UKERC Energy Data Centre, Electricity Association, Elexon Ltd., 2013. Electricity user load profiles by profile class. Tech. rep., Electricity Association, London, United Kingdom.

URL http://data.ukedc.rl.ac.uk/browse/ edc/Electricity/LoadProfile/doc

[27] Vassileva, I., Wallin, F., Dahlquist, E., feb 2012. Analytical comparison between electricity consumption and behavioral characteristics of
Swedish households in rented apartments. Applied Energy 90 (1), 182-188.

URL http://doi.org/10.1016/J.APENERGY. 2011.05 .031

[28] Widén, J., Wäckelgård, E., 2010. A highresolution stochastic model of domestic activity patterns and electricity demand. Applied Energy 87 (6), 1880-1892.

URL https://doi.org/10.1016/j. apenergy .2009.11.006

[29] Widén, J., Wäckelgård, E., jun 2010. A highresolution stochastic model of domestic activity patterns and electricity demand. Applied Energy 87 (6), 1880-1892.

URL https://doi.org/10.1016/j. apenergy .2009.11.006

[30] Wilke, U., Haldi, F., Scartezzini, J.-L., Robinson, D., feb 2013. A bottom-up stochastic model to predict building occupants' time-dependent activities. Building and Environment 60, 254264.

URL https://doi.org/10.1016/j. buildenv.2012.10.021

[31] Yao, R., Steemers, K., 2005. A method of formulating energy load profile for domestic buildings in the UK. Energy and Buildings 37 (6), 663671 .

URL https://doi.org/10.1016/j.enbuild. 2004.09 .007

[32] Zimmermann, J.-P., Evans, M., Griggs, J., King, N., Harding, L., Roberts, P., Evans, C., 2012. Household Electricity Survey: A study of domestic electrical produc usage - Final report. Tech. rep., Intertek, London, United Kingdom. 